dase may provide a new therapeutic avenue to block allergic inflammation at its earliest known step and help those of us who live in the pollen belt to better appreciate the arrival of spring.

Address correspondence to: J. David Lambeth, Department of Pathology and Laboratory Medicine, 615 Michael Street, Emory University School of Medicine, Atlanta, Georgia 30322, USA. Phone: (404) 727-5875; Fax: (404) 727-2783; E-mail: noxdoc@mac.com.

1. National Institute of Allergy and Infectious Disease. 2003. Airborne allergens: something in the air. National Institutes of Health. NIH Publication No. 03-7045. http://www.niaid.nih.gov/publications/allergens/airborne_allergens.pdf.

2. Herrick, C.A., and Bottomly, K. 2003. To respond or not to respond: T cells in allergic asthma. Nat. Rev. Immunol. 3:405-412.
3. Bousquet, J., et al. 1990. Eosinophilic inflammation in asthma. N. Engl. J. Med. 323:1033-1039.

4. Bowler, R.P., and Crapo, J.D. 2002. Oxidative stress in allergic respiratory diseases. J. Allergy Clin. Immunol. 110:349-356.

5. Andreadis, A.A., Hazen, S.L., Comhair, S.A.A., and Erzurum, S.C. 2003. Oxidative and nitrosative events in asthma. Free Radic. Biol. Med. 35:213-225.

6. Babior, B.M., Lambeth, J.D., and Nauseef, W. 2002. The neutrophil NADPH oxidase. Arch. Biochem. Biophys. 397:342-344.

7. Vignais, P.V. 2002. The superoxide-generating NADPH oxidase: structural aspects and activation mechanism. Cell. Mol. Life Sci. 59:1428-1459.

8. Lambeth, J.D. 2002. Nox/Duox family of nicotinamide adenine dinucleotide (phosphate) oxidases. Curr. Opin. Hematol. 9:11-17.

9. Boldogh, I., et al. 2005. ROS generated by pollen NADPH oxidase provide a signal that augments antigen-induced allergic airway inflammation. J. Clin. Invest. 115:2169-2179. doi:10.1172/JCI24422.

10. Suh, Y.A., et al. 1999. Cell transformation by the superoxide-generating oxidase Mox1. Nature. 401:79-82.

11. Lambeth, J.D., Cheng, G., Arnold, R.S., and Edens, W.A. 2000. Novel homologs of gp91phox. Trends Biochem. Sci. 25:459-461.
12. Lambeth, J.D. 2004. NOX enzymes and the biology of reactive oxygen. Nat. Rev. Immunol. 4:181-189.

13. Heyworth, P.G., Cross, A.R., and Curnutte, J.T. 2003. Chronic granulomatous disease. Curr. Opin. Immunol. 15:578-584.

14. Banfi, B., et al. 2004. NOX3, a superoxide-generating NADPH oxidase of the inner ear. J. Biol. Chem. 279:46065-46072.

15. Paffenholz, R., et al. 2004. Vestibular defects in headtilt mice result from mutations in Nox3, encoding an NADPH oxidase. Genes Dev. 18:486-491.

16. Moreno, J.C., et al. 2002. Inactivating mutations in the gene for thyroid oxidase 2 (THOX2) and congenital hypothyroidism. N. Engl. J. Med. 347:95-102.

17. Lamb, C., and Dixon, R.A. 1997. The oxidative burst in plant disease resistance. Annu. Rev. Plant Physiol. Plant Mol. Biol. 48:251-275.

18. Kwak, J.M., et al. 2003. NADPH oxidase AtrbohD and AtrbohF genes function in ROS-dependent ABA signaling in Arabidopsis. EMBO J. 22:2623-2633.

19. Foreman, J., et al. 2003. Reactive oxygen species produced by NADPH oxidase regulate plant cell growth. Nature. 422:442-446.

20. Feijo, J.A., Costa, S.S., Prado, A.M., Becker, J.D., and Certal, A.C. 2004. Signalling by tips. Curr. Opin. Plant Biol. 7:589-598.

\title{
Insulin infusion in acute illness
}

\section{Paresh Dandona, Priya Mohanty, Ajay Chaudhuri, Rajesh Garg, and Ahmad Aljada}

Division of Endocrinology, Diabetes and Metabolism, State University of New York at Buffalo, and Kaleida Health, Buffalo, New York, New York, USA.

\begin{abstract}
The discovery of the antiinflammatory effect of insulin and the proinflammatory effect of glucose has not only provided novel insight into the mechanisms underlying several disease states but has also provided a rationale for the treatment of hyperglycemia in several acute clinical conditions. Van den Berghe et al. previously showed the benefits of intensive glycemic control with insulin in patients admitted to intensive care units. In this issue of the JCI, the same group of investigators now demonstrates that infusion of insulin to restore euglycemia in these patients results in a marked reduction in inflammatory indices such as adhesion molecules, hepatic iNOS, and plasma NO metabolites (see the related article beginning on page 2277). The reduction in the mediators of inflammation may thus be responsible for the impressive improvement in clinical outcomes following insulin therapy, and the results suggest a new paradigm in which glucose and insulin are related not only through their metabolic actions but also through their opposite effects on inflammatory mechanisms.
\end{abstract}

The importance of hyperglycemia in the pathogenesis and prognosis of acute illness and the benefit of controlling glucose with insulin in the critically ill hospitalized patient have emerged over the past 4 years. Three sets of data appeared almost simultaneously, which drew attention to this issue.

Nonstandard abbreviations used: AP-1, activator protein-1; EGR-1, early growth response-1; ICU, intensive care unit; TF, tissue factor.

Conflict of interest: Paresh Dandona is a consultant and speaker for Sanofi-Aventis and Novo Nordisk.

Citation for this article: J. Clin. Invest. 115:2069-2072 (2005). doi:10.1172/JCI26045.
First, glucose and lipid administration were shown to result in oxidative and inflammatory stress (1-3). Second, insulin, the hormone secreted in response to glucose and macronutrient intake, was found to suppress ROS generation and the activation of inflammatory mechanisms $(4,5)$. Thus, glucose was shown to be proinflammatory while insulin is antiinflammatory (Figure 1). Third, and almost simultaneously with the 2 previous studies, came the observation that the restoration of normoglycemia by insulin infusion in hyperglycemic patients in a surgical intensive care unit (ICU) resulted in a $50 \%$ reduction in mortality along with several other benefits, including a reduction in the incidence of renal failure (and thus the need for dialysis), septicemia, and ICU neuropathy (6). The need for blood transfusion and the duration of ventilation also fell significantly in these patients. These effects of insulin infusion in acutely ill patients were remarkable and totally unexpected. This large, landmark study provided clinical evidence that the proinflammatory effects of glucose and antiinflammatory effects of insulin probably play an important role in determining clinical outcome in acute illness. In this same study (6), the clinical benefits were associated with a fall in C-reactive protein level (7). A close relationship was found between the fall in blood glucose concentration and the improvement in clinical outcome.

Overall, the data emphasized that hyperglycemia is potentially toxic (6). This is consistent with a proinflammatory effect of glucose. In a subsequent publication, it was shown that there were marked mitochondrial abnormalities in the hepatocytes of the control group, which were prevented in the insulin infusion group with the restoration of normoglycemia (8). The mitochondrial abnormalities were char- 


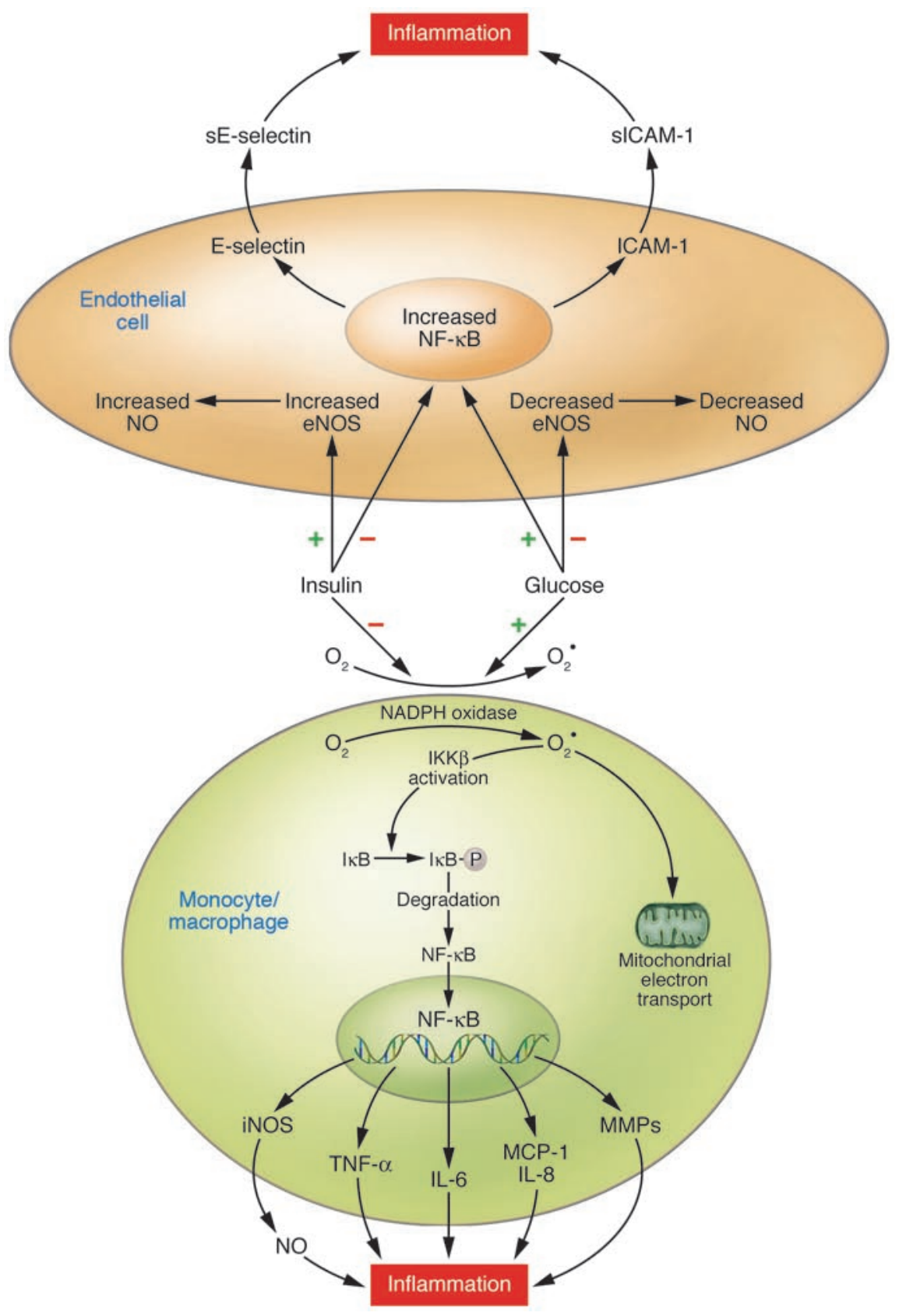

acterized by marked swelling of the mitochondria, distortion of the cristae, and the separation of the inner mitochondrial membrane from the outer one. There was also an abnormality of the enzymes in the electron transport chain. The authors suggested that increased ROS generation and oxidative stress induced these abnormalities. This is consistent with the ROS-suppressive effect of insulin.

\section{Mechanism of insulin action}

Insulin suppresses 3 major proinflammatory transcription factors: NF- $\kappa \mathrm{B}$, activa-

\section{Figure 1}

The antiinflammatory effect of insulin and the proinflammatory effect of glucose. Insulin suppresses $\mathrm{ROS}$ and $\mathrm{O}_{2} \cdot$ generation and NADPH oxidase expression, while glucose stimulates both. Within the macrophage, $\mathrm{O}_{2}{ }^{\circ}$ activates inhibitor of NF-KB kinase $\beta$ (IKK $\beta$ ) to enhance phosphorylation of $I_{\kappa} B(\alpha$ and $\beta$ ) such that it undergoes proteasomal degradation, releasing NF-кB to translocate into the nucleus. $\mathrm{NF}-\kappa \mathrm{B}$ stimulates the transcription of genes encoding proinflammatory proteins including TNF- $\alpha$, IL-6, monocyte chemoattractant protein 1 (MCP-1), and MMPs. Within the endothelial cell, insulin also induces eNOS expression in endothelial cells, which leads to controlled NO release and vasodilation, while glucose has the opposite effect. Glucose induces the expression of adhesion molecules ICAM-1 and E-selectin, while insulin suppresses their expression in the endothelial cell. sE-selectin, soluble E-selectin; sICAM-1, soluble ICAM-1.

help stabilize the plaque. MMP-1 is known to activate protease-activated receptor-1, which mediates the action of thrombin and thus triggers prothrombotic and proconstrictor processes, which will also be potentially suppressed by insulin. In addition, insulin suppresses ROS generation and the expression of $\mathrm{p}^{4} 7^{\mathrm{phox}}$, a key component of NADPH oxidase, the enzyme that generates the superoxide radical, consistent with the potent antioxidant effect of insulin $(4,9)$. Two other important effects of insulin include vasodilatation and the inhibition of platelet aggregation (10-12). These effects are mediated by an increase in NO release and NO synthase activity in the endothelium and the platelet $(13,14)$. Platelet inhibition may also contribute to insulin's antiinflammatory effect, since platelets are loaded with CD40 ligand, serotonin, and histamine.

\section{Glucose: an inducer of oxidative stress and inflammation}

Glucose, on the other hand, is proinflammatory, and even a 75-g glucose load given orally to normal subjects results in profound oxidative stress and inflammatory changes at the cellular and molecular level. This occurs even without an increase in plasma glucose concentrations into the pathological range and in spite of endogenous insulin secretion $(1,3)$. Therefore, if high plasma glucose concentrations are maintained, they can be expected to be profoundly proinflammatory. This is indeed the case, especially if endogenous insulin 
secretion is inhibited (15). Not only does glucose induce increased intranuclear NF- $\mathrm{KB}$ binding, it induces increased AP-1 and EGR-1 binding. Thus, glucose activates all 3 key proinflammatory transcription factors suppressed by insulin $(3,16)$ (Figure 1). Generation of cytokines (e.g., TNF- $\alpha$ ) by mononuclear cells is stimulated in addition to an increase in MMP- 2 and TF protein expression following the ingestion of glucose. In addition, plasma MMP-2, MMP-9, and TF concentrations increase (16). Glucose also induces an increase in ROS (including superoxide radical) generation and an increase in $\mathrm{p} 47 \mathrm{phox}$ expression, which is consistent with an increase in NADPH oxidase (1). The bioavailability of $\mathrm{NO}$ is reduced due to the increase in the level of superoxide radical, which binds NO to form peroxynitrite. This exerts a proconstrictor, platelet proaggregatory, and prothrombotic effect. Through the induction of MMPs, glucose may promote plaque rupture and thus trigger thrombosis. Thus, hyperglycemia induces a milieu of oxidative stress, inflammation, vascular constriction, platelet hyperaggregability, and thrombosis. The proinflammatory and prothrombotic effects of glucose are profound.

\section{The future of insulin infusions}

Inflammation is a normal physiological defense mechanism. However, in severe inflammatory conditions, the excessive nature of this defense mechanism can contribute to tissue damage. Thus, its control could potentially improve clinical outcomes. Glucose is proinflammatory, and insulin is the ideal antiinflammatory measure, since it normalizes plasma glucose concentrations while exerting its antiinflammatory effect. In addition, insulin, as an anabolic hormone, could help control the catabolic state induced by severe inflammation. Thus, insulin infusion could potentially benefit conditions with severe systemic inflammation such as septicemia, meningitis, and pneumonias. The use of insulin infusions to maintain normoglycemia has already been shown to be of benefit during cardiac surgery (17), in patients with burns (18), and in those in medical ICUs (19). Furthermore, the antiinflammatory effect of insulin has been confirmed in rats injected with endotoxin (20) and in patients with severe forms of hyperglycemia including diabetic ketoacidosis (21). Similarly, insulin infusion has been shown to be antiinflammatory and cardioprotective in acute myocardial infarction (9).
A new study by Langouche, Van den Berghe et al., reported in this issue of the JCI (22), on ICU patients from the same cohort in which the authors previously established markedly improved survival (6), further emphasizes the role of insulin infusion in suppression of inflammation. Here they demonstrate, as might be expected, a suppression of several mediators of inflammation such as the adhesion molecules ICAM-1 and E-selectin (Figure 1). In addition, these patients had markedly elevated plasma $\mathrm{NO}\left(\mathrm{NO}_{2}+\mathrm{NO}_{3}\right)$ concentrations as well as an increase in iNOS expression in the liver. Both plasma NO concentrations and iNOS expression were reduced by insulin, while eNOS expression was not altered. This very interesting observation implies that the source of the excessive NO is probably the iNOS expressed by macrophages in the liver (Kupffer cells). It is possible that the reticuloendothelial system elsewhere also contributes to this phenomenon. Insulin infusion likely suppresses iNOS expression not just in the liver but elsewhere. It is even more remarkable that the NO concentration predicted mortality: patients in the 2 highest quartiles of NO concentration had a risk ratio of 8.0 and 6.0, respectively, when compared with patients in the lowest quartile. This makes NO concentration the strongest risk factor for mortality in the ICU. Although the data on iNOS expression was obtained from the patients who died and therefore may not be precise, the marked suppression of iNOS, by $66 \%$, following insulin infusion is consistent with a fall in NO. In contrast, eNOS levels did not change. This indicates that the contribution to the increase in $\mathrm{NO}$ concentration in these patients or the suppression of $\mathrm{NO}$ in those infused with insulin was not due to endothelial NO but due to iNOS from monocytes/macrophages or other cells in which iNOS has been induced as a result of inflammation (Figure 1). These data offer us the opportunity to use NO concentration as a prognostic marker and as an important mediator of the pathological process in ICU patients.

The data also demonstrate a small (15\%) but significant reduction in ICAM-1, an adhesion molecule essential to the inflammatory response (22). The magnitude of this reduction is not likely to be responsible for the remarkable improvement in clinical outcomes, although it is consistent with an improvement in endothelial function.

More and more evidence of the antiinflammatory effect of insulin continues to emerge. The demonstration of its antiinflammatory action 80 years after its discovery as a metabolic hormone and more than 25 years after the discovery of the insulin receptor in the mononuclear cell (including the monocyte/macrophage), a key mediator of inflammation, is a major advance. Similarly, the emergence of glucose as an inducer of oxidative and inflammatory stress and a prognostic indicator of grave clinical outcomes offers important potential therapeutic opportunities. The outstanding studies discussed here represent significant advances in this rapidly developing field, and they support a new paradigm in which glucose and insulin are related not only through their metabolic action but also through inflammatory mechanisms.

Address correspondence to: Paresh Dandona, Diabetes-Endocrinology Center of Western New York, 3 Gates Circle, Buffalo, New York 14209, USA. Phone: (716) 887-4523; Fax: (716) 887-4773; E-mail: pdandona@kaleidahealth.org.

1. Mohanty, P., et al. 2000. Glucose challenge stimulates reactive oxygen species (ROS) generation by leucocytes. J. Clin. Endocrinol. Metab. 85:2970-2973.

2. Tripathy, D., et al. 2003. Elevation of free fatty acids induces inflammation and impairs vascular reactivity in healthy subjects. Diabetes. 52:2882-2887.

3. Dhindsa, S., et al. 2004. Differential effects of glucose and alcohol on reactive oxygen species generation and intranuclear nuclear factor-kappaB in mononuclear cells. Metabolism. 53:330-334.

4. Dandona, P., et al. 2001. Insulin inhibits intranuclear nuclear factor kappaB and stimulates IkappaB in mononuclear cells in obese subjects: evidence for an anti-inflammatory effect? J. Clin. Endocrinol. Metab. 86:3257-3265.

5. Aljada, A., Ghanim, H., Mohanty, P., Kapur, N., and Dandona, P. 2002. Insulin inhibits the pro-inflammatory transcription factor early growth response gene-1 (Egr)-1 expression in mononuclear cells (MNC) and reduces plasma tissue factor (TF) and plasminogen activator inhibitor-1 (PAI-1) concentrations. J. Clin. Endocrinol. Metab. 87:1419-1422.

6. Van den Berghe, G., et al. 2001. Intensive insulin therapy in the critically ill patients. N. Engl. J. Med. 345:1359-1367.

7. Hansen, T.K., Thiel, S., Wouters, P.J., Christiansen, J.S., and Van den Berghe, G. 2003. Intensive insulin therapy exerts antiinflammatory effects in critically ill patients and counteracts the adverse effect of low mannose-binding lectin levels. J. Clin. Endocrinol. Metab. 88:1082-1088.

8. Vanhorebeek, I., et al. 2005. Protection of hepatocyte mitochondrial ultrastructure and function by strict blood glucose control with insulin in critically ill patients. Lancet. 365:53-59.

9. Chaudhuri, A., et al. 2004. Anti-inflammatory and profibrinolytic effect of insulin in acute ST-segment-elevation myocardial infarction. Circulation. 109:849-854

10. Grover, A., et al. 1995. Insulin attenuates norepinephrine-induced venoconstriction. An ultrasonographic study. Hypertension. 25:779-784.

11. Steinberg, H.O., Brechtel, G., Johnson, A., Fineberg, N., and Baron, A.D. 1994. Insulin-mediated skel- 
etal muscle vasodilation is nitric oxide dependent. A novel action of insulin to increase nitric oxide release. J. Clin. Invest. 94:1172-1179.

12. Trovati, M., et al. 1994. Insulin increases guanosine$3^{\prime}, 5^{\prime}$-cyclic monophosphate in human platelets. A mechanism involved in the insulin anti-aggregating effect. Diabetes. 43:1015-1019.

13. Aljada, A., and Dandona, P. 2000. Effect of insulin on human aortic endothelial nitric oxide synthase. Metabolism. 49:147-150.

14. Zeng, G., and Quon, M.J. 1996. Insulin-stimulated production of nitric oxide is inhibited by wortmannin. Direct measurement in vascular endothelial cells. J. Clin. Invest. 98:894-898.

15. Esposito, K., et al. 2002. Inflammatory cytokine concentrations are acutely increased by hyperglyce- mia in humans: role of oxidative stress. Circulation. 106:2067-2072.

16. Aljada, A., et al. 2004. Glucose intake induces an increase in activator protein 1 and early growth response 1 binding activities, in the expression of tissue factor and matrix metalloproteinase in mononuclear cells, and in plasma tissue factor and matrix metalloproteinase concentrations. Am. J. Clin. Nutr. 80:51-57.

17. Furnary, A.P., et al. 2003. Continuous insulin infusion reduces mortality in patients with diabetes undergoing coronary artery bypass grafting. J. Thorac. Cardiovasc. Surg. 125:1007-1021.

18. Jeschke, M.G., Klein, D., and Herndon, D.N. 2004. Insulin treatment improves the systemic inflammatory reaction to severe trauma. Ann. Surg.
239:553-560

19. Krinsley, J.S. 2004. Effect of an intensive glucose management protocol on the mortality of critically ill adult patients. Mayo Clin. Proc. 79:992-1000.

20. Jeschke, M.G., Klein, D., Bolder, U., and Einspanier, R. 2004. Insulin attenuates the systemic inflammatory response in endotoxemic rats. Endocrinology. 145:4084-4093.

21. Stentz, F.B., Umpierrez, G.E., Cuervo, R., and Kitabchi, A.E. 2004. Proinflammatory cytokines, markers of cardiovascular risks, oxidative stress, and lipid peroxidation in patients with hyperglycemic crises. Diabetes. 53:2079-2086

22. Langouche, L., et al. 2005. Intensive insulin therapy protects the endothelium of critically ill patients. J. Clin. Invest. 115:2277-2286. doi:10.1172/JCI25385.

\title{
You are right too!
}

\author{
Joseph L. Witztum
}

Department of Medicine, University of California San Diego, La Jolla, California, USA.

\begin{abstract}
Macrophage scavenger receptors, such as CD36 and class A scavenger receptor (SR-A), have previously been thought to play a central role in foam cell formation and atherogenesis by mediating the uptake of oxidized LDL. In this issue of the JCI, Moore et al. report that $\mathrm{Apoe}^{-/-}$mice deficient in either CD36 or SR-A did not have less atherosclerosis at the level of the aortic valve than did wild-type $A p o e^{-/-}$mice (see the related article beginning on page 2192). In contrast, similar studies by previous investigators found that deletion of these receptors decreased atherogenesis. The reasons for the different results are not known, but these data suggest that the role of these receptors in atherogenesis remains unresolved.
\end{abstract}

In the small villages of eastern Europe, the rabbi was the undisputed leader of the Jewish people of his village. Not only was he the master of religious wisdom and law, but he often served as the arbiter of civil disputes as well. There is told the tale of 2 individuals who had a major dispute and agreed to go to the rabbi for resolution. The first party to the dispute came to the rabbi and carefully outlined his side of the argument. The rabbi listened intently and finally said, "My friend, you are right." The man went away satisfied. Later in the day, the other party to the dispute arrived and told the rabbi his side of the issue. The rabbi again listened carefully, was impressed with the arguments, and replied after some thought, "You are right." Later, the rabbi's wife, who had overheard the rabbi's conversations with both men, said to him, "Rabbi, you

Nonstandard abbreviations used: LDLR, LDL receptor; mmLDL, minimally modified LDL; OxLDL, oxidized LDL; SR-A, class A scavenger receptor; SR-BI, class B, type I scavenger receptor.

Conflict of interest: The author has declared that no conflict of interest exists.

Citation for this article: J. Clin. Invest. 115:2072-2075 (2005). doi:10.1172/JCI26130. told both the first party and the second party that they were right. How can this be?" To which the rabbi replied, "And you are right too!"

So we too are faced with a dilemma. In the current issue of the JCI, Moore et al. report that $A p^{-/-}$mice deficient in either class A scavenger receptor (SR-A) or CD36 and fed an atherogenic diet showed a reduction in peritoneal macrophage lipid accumulation in vivo; however, there was no decrease in atherogenesis (1). To put these results into perspective, previous reports by equally reputable investigators have provided convincing data supporting a role for these 2 scavenger receptors in the generation of foam cells and the development of atherosclerosis (2-4). These data are particularly strong for the CD36 receptor. Furthermore, a recent study in which bone marrow from either $\mathrm{Cd} 36^{-/-}$or wildtype mice was transplanted into cholesterol-fed Apoe-/- mice demonstrated that the absence of CD36 provided substantial protection against lesion formation and the reintroduction of $\mathrm{CD} 36^{+}$macrophages increased lesion area (5). The data strongly suggest that CD36 expressed on macrophages is an important contributor to foam cell formation and atherosclerosis. So like the rabbi's wife, we are left to ask, "How can this be?"

\section{Analysis of the study}

Let us first examine the current study by Moore et al. (1). There are many aspects of their data that support their conclusions. The authors have generated a carefully defined genetic model that was essentially congenic to the $\mathrm{C} 57 \mathrm{BL} / 6$ background. The authors began with the same $\mathrm{Sra}^{-/-}$mice generated by Kodama and colleagues (2) and the $C d 36^{-/-}$mice generated by Febbraio and Silverstein (4) and backcrossed them to be $99 \%$ congenic with the C57BL/ 6 background. In this respect, the mice studied by Moore et al. did differ from some, but not all, of the animals used in similar experiments. It is of course well known that different murine strains have differing susceptibilities to atherosclerosis and, as even a single gene product can influence this susceptibility, it is conceivable that some of the prior experiments were influenced by the presence of unrecognized diseasemodifying gene(s) that were not a factor in the experiments presented here (1). However, the results of the bone marrow transplant study cited above (5) suggest that if such disease-modifying genes are involved, they would need to be primary products of bone marrow-derived cells.

Despite the robustness of the observation that atherosclerosis was not decreased at the aortic valves in both $\mathrm{Sra}^{-/-} \mathrm{Apoe}^{-/-}$and $\mathrm{Cd} 36^{-/-} \mathrm{Apoe}^{-/-}$mice, a number of issues need to be considered in interpreting these 